\title{
Dynamic Electrocardiographic Changes due to Cardiac Compression by a Giant Hiatal Hernia
}

\author{
Jun HoKamaki, Hiroaki KaWAno, Shinzo MiYamoto, Seigo SugiYama, \\ Ryuichiro FuKushima, Tomohiro SaKamoto, Michihiro Yoshimura and Hisao Ogawa
}

\begin{abstract}
A 79-year-old woman was admitted due to chest pain with $\mathbf{T}$ wave inversion and anasarca. Echocardiography demonstrated a mass compressing the heart and computed tomography revealed a giant hiatal hernia within the intrathoracic stomach located just behind the heart. After drainage of the gastric contents, the $T$ wave inversion disappeared, but subsequent ST elevation in leads $V_{1}-V_{6}$ was noted. After surgical correction of the hiatal hernia, the ST segment elevation returned to a nearly normal level. The changes in the compressed heart induced by hiatal hernia may cause pericarditis resulting in electrocardiographic changes.
\end{abstract}

(Internal Medicine 44: 136-140, 2005)

Key words: giant hiatal hernia, electrocardiographic changes, edema

\section{Introduction}

Hiatal hernia is a relatively common condition in the general population. Most of the patients with hiatal hernia are asymptomatic. In symptomatic patients, hiatal hernia is generally associated with gastroesophageal reflux with symptoms of heartburn or chest pain. Thus, hiatal hernia sometimes leads to misdiagnosis of ischemic heart disease, reflux esophagitis, and gastritis $(1,2)$. Moreover, gastro-pericardial fistula $(3,4)$, arrhythmia $(5,6)$, and heart failure (7) have been reported as cardiac complications of hiatal hernia in some cases. However, few previous reports demonstrate the time course of electrocardiographic changes in patients with a hiatal hernia. We document the time course of electrocardiographic changes in a patient with a giant hiatal hernia.

\section{Case Report}

A 79-year-old woman was referred to our hospital after having symptoms of sudden-onset chest pain and vomiting. She had been diagnosed with hypothyroidism 10 years previously. In spite of taking levothyroxine, she still had remarkable general edema. Moreover, she had exertional dyspnea for the past 10 years. She had no history of myocardial infarction or angina pectoris, and there was no family history of ischemic heart disease. She suddenly experienced severe anterior chest pain at rest and was brought to the nearest hospital by ambulance. However, the persistence of her chest pain together with vomiting and cold sweat led the patient to consult our hospital with the suspicion of acute coronary syndrome (ACS).

On admission, the patient was $144.9 \mathrm{~cm}$ tall and weighed $57.5 \mathrm{~kg}$. Her blood pressure was $149 / 79 \mathrm{mmHg}$, and her pulse rate was 60 beats per minutes (bpm) with a regular sinus rhythm. A grade 2/6 holosystolic murmur was audible at the apex. The patient was evidently tachypneic. Findings on physical examination were significant facial and leg edema, and remarkable epigastric tenderness. The jugular veins were markedly distended, and showed Kussmaul's sign in the semi-Fowler position. The hematological examination showed a white blood cell count of $2,800 / \mu 1$. Laboratory tests revealed hyperglycemia (glucose $170 \mathrm{mg} / \mathrm{dl}$ ), elevated serum concentrations of total bilirubin $(1.2 \mathrm{mg} / \mathrm{dl})$, lactate dehydrogenase $(265 \mathrm{U} / l)$, C-reactive protein (CRP, 0.56 $\mathrm{mg} / \mathrm{dl}$ ), and brain natriuretic peptide (BNP, $123 \mathrm{pg} / \mathrm{ml}$ ). Serum electrolyte values were as follows: sodium $145 \mathrm{mEq} /$ $l$, potassium $3.5 \mathrm{mEq} / l$, chloride $107 \mathrm{mEq} / l$, calcium 8.4 $\mathrm{mg} / \mathrm{dl}$. A specimen of arterial blood revealed that the $\mathrm{pH}$ was 7.416 , and the bicarbonate was $28.6 \mathrm{mmol} / l$. The plasma concentrations of thyroid hormones were normal.

The chest X-ray showed a large abnormal shadow overlapping the heart (Fig. 1). The electrocardiogram demonstrated sinus rhythm at a rate of $64 \mathrm{bpm}, \mathrm{T}$ wave inversion in

From the Department of Cardiovascular Medicine, Graduate School of Medical Sciences Kumamoto University, Kumamoto

Received for publication April 7, 2004; Accepted for publication October 1, 2004

Reprint requests should be addressed to Dr. Hiroaki Kawano, the Department of Cardiovascular Medicine, Graduate School of Medical Sciences Kumamoto University, 1-1-1 Honjo, Kumamoto 860-8556 


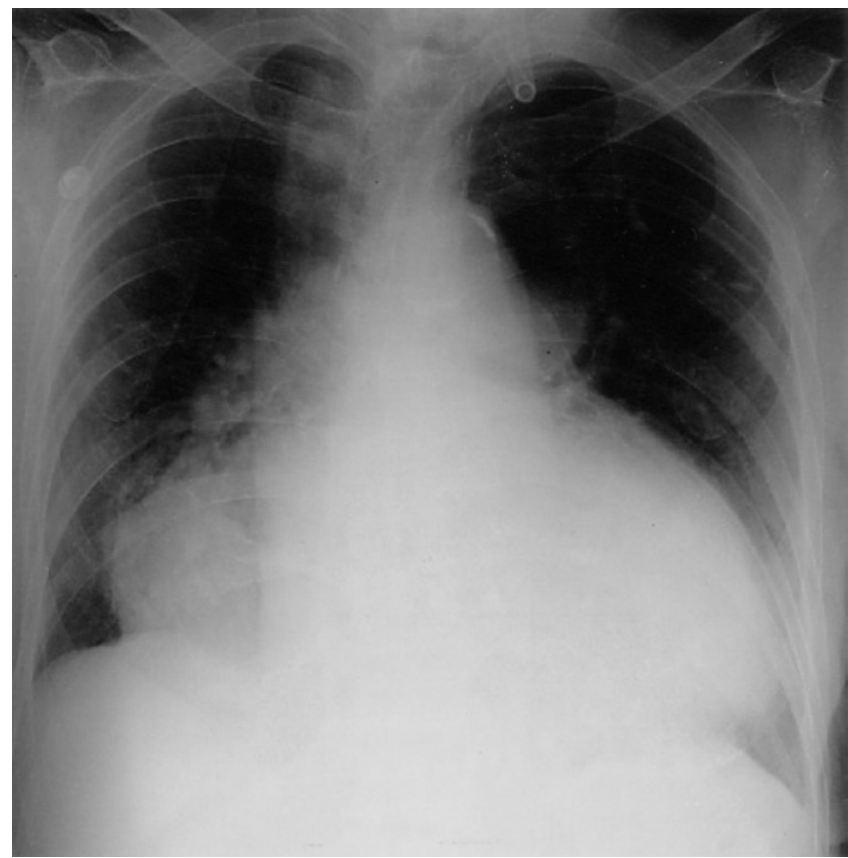

Figure 1. Chest X-ray. A large abnormal shadow overlapping the heart. leads I, II, III, ${ }_{\mathrm{a}} \mathrm{V}_{\mathrm{L}}, \mathrm{V}_{\mathrm{F}}$ and $\mathrm{V}_{3}-\mathrm{V}_{6}$, and a prolonged QTc interval (Fig. 2B). We administrated famotidine $(20 \mathrm{mg})$ intravenously at the time of admission. However, she was not receiving any other drugs that might have prolonged the QTc interval. Transthoracic echocardiography revealed a mass that compressed the left atrium, left ventricle, and inferior vena cava (IVC), but the cause of the mass was not identified (Fig. 3A). The wall motion of the left ventricle showed normal contraction without asynergy. In addition, moderate tricuspid regurgitation was observed, and there was no significant pericardial effusion.

Computed tomography (CT) was performed in order to determine the cause of the mass. CT revealed a type III giant hiatal hernia within the intrathoracic stomach located just behind the heart with resultant compression of the heart and IVC. Moreover, most of the stomach had migrated into the thoracic cavity (Fig. 4).

The drainage of intragastric fluid relieved her symptoms. However, the electrocardiogram showed ST elevation in leads I, II, III, ${ }_{a} V_{F}$, and $V_{1}-V_{6}$ (Fig. 2C). We then performed transthoracic echocardiography immediately to evaluate ventricular wall motion. However, echocardiography did not show any asynergy of the left ventricle, but did show release of the IVC compression (Fig. 3B). Based on the echocardiographic findings, it was felt that the chest pain and electrocardiographic changes were not due to ACS, but due to the
A

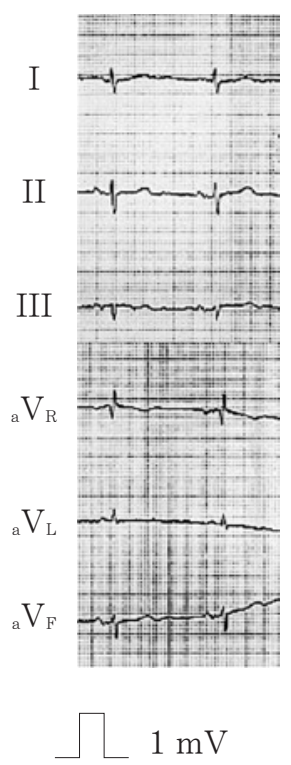

Before admission
B

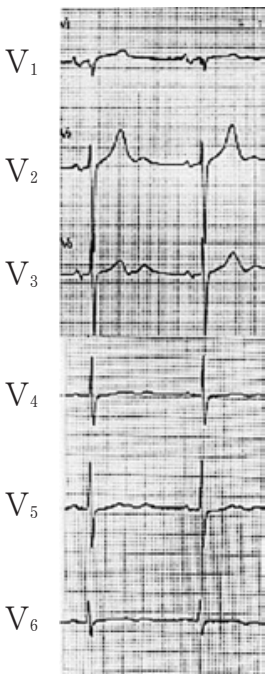

On admission

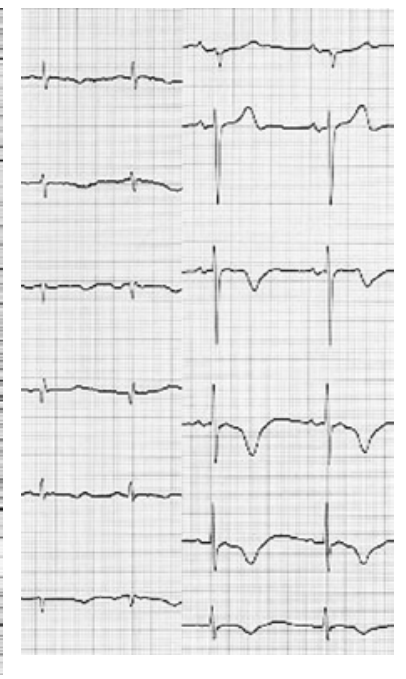

After drainage (3 hours after admission)
C

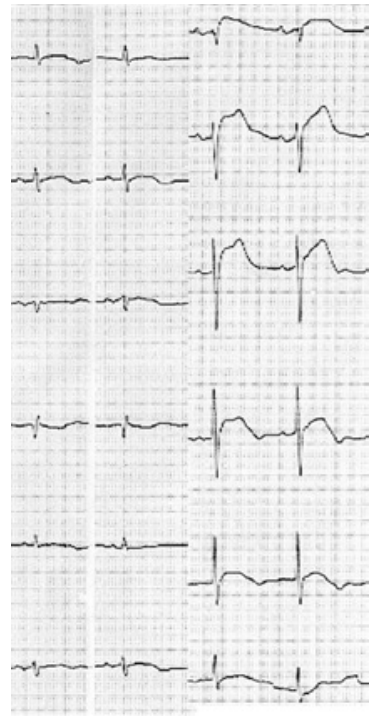

.

After operation

(3 weeks after admission)

Figure 2. Time course of changes observed in the electrocardiogram. Electrocardiogram before admission (A) showed prominent $U$ wave in lead $V_{3}$. However, the electrocardiogram did not indicate $T$ wave inversion. Electrocardiogram on admission (B) showed $T$ wave inversion in leads I, II, III, ${ }_{a} V_{L},{ }_{a} V_{F}$ and $V_{3}-V_{6}$, and a prolonged QTc interval. After drainage of the gastric contents, elevation of the ST segment appeared in leads I, II, III, ${ }_{a} V_{F}$ and $V_{1}-V_{6}(C)$. The ST segment level, $T$ wave amplitude and QTc interval had returned to almost normal after the operation (D). 
A
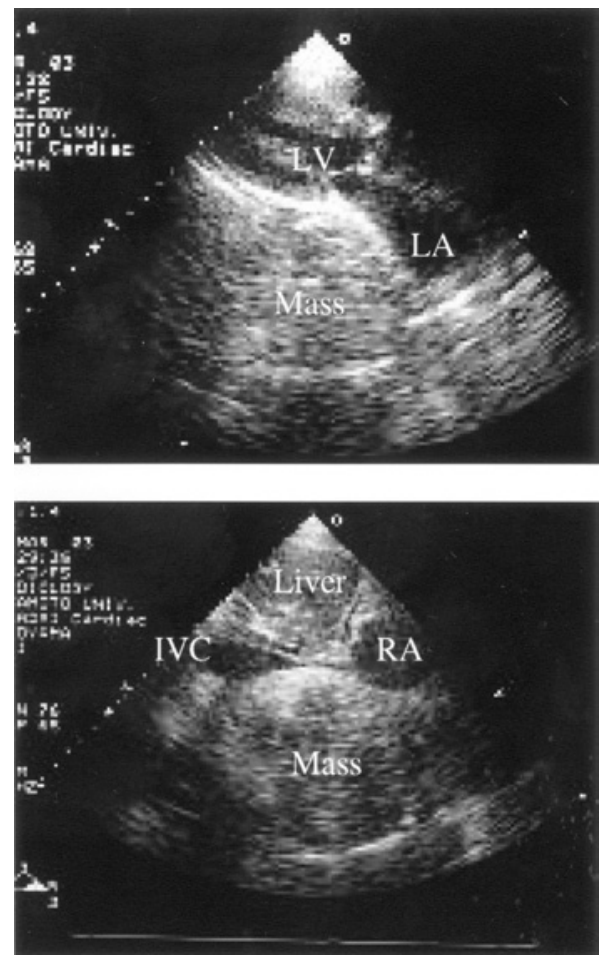

On admission
B


After drainage
C



After operation

Figure 3. Results of serial echocardiography. A giant hiatal hernia showed compression of the heart and inferior vena cava (A). The compression of the inferior vena cava was relieved, and pericardial effusion appeared after drainage (3 days after admission) (B). Pericardial effusion vanished after operation (C).
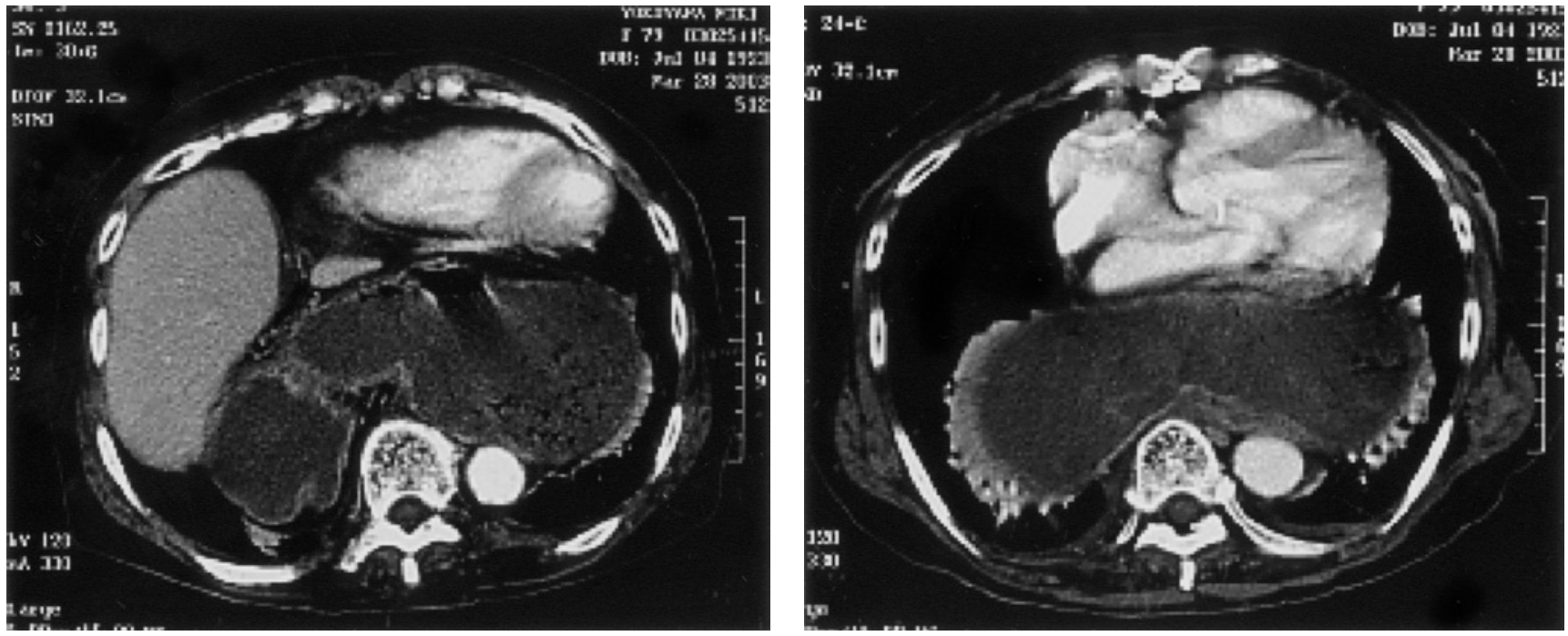

Figure 4. Computed tomography. Computed tomography scan revealed a giant hiatal hernia with intrathoracic stomach located just behind the heart with resultant compression of the heart and inferior vena cava. Most of the stomach migrated into the thoracic cavity. 
giant hiatal hernia and obstruction of the stomach. Thus, the patient received an operation for the hiatal hernia 2 weeks after admission (floppy nissen fundoplication, Hill's method).

The electrocardiogram before admission showed a positive or flat $\mathrm{T}$ wave in leads I, II, III, ${ }_{\mathrm{a}} \mathrm{V}_{\mathrm{L}},{ }_{\mathrm{a}} \mathrm{V}_{\mathrm{F}}$ and $\mathrm{V}_{3}-\mathrm{V}_{6}$, and prominent $\mathrm{U}$ wave in lead $\mathrm{V}_{3}$ (Fig. 2A). The ST segment elevation, $\mathrm{T}$ wave inversion and QTc prolongation were almost completely resolved after the operation (Fig. 2D). Echocardiography 3 days after drainage showed pericardial effusion (Fig. 3B). Her white blood cell count $(4,000 / \mu \mathrm{l})$ and CRP level $(1.82 \mathrm{mg} / \mathrm{dl})$ peaked on the day after drainage. The pericardial effusion vanished (Fig. 3C) and the facial and leg edema improved after the operation to correct the hernia (3 weeks after admission).

\section{Discussion}

To the best of our knowledge, this is the first clinical report of the time course of electrocardiographic changes in a patient with a giant hiatal hernia. The patient had chest pain, facial and leg edema, and $\mathrm{T}$ wave inversion on admission. ST elevation similar to that observed in pericarditis occurred when the cardiac compression was reduced by the drainage of the intragastric fluid. After the operation for the hiatal hernia, compression of the heart was relieved and the elevated ST segment returned to normal. Moreover, her facial and leg edema improved. Thus, it is possible that these conditions might be produced as a change of direct or indirect pressure exerted on the heart and IVC.

Type III hiatal hernia is composed of the combination of sliding and paraesophageal hernia components. Patients with this combined hernia will often have symptoms related to pathologic gastroesophageal reflux and to mechanical obstruction of the stomach within the paraesophageal components of the hernia. In the present case, we thought that the chest pain was related to mechanical obstruction of the stomach. Furthermore, Ito and co-workers reported that severe exertional dyspnea results from a large hiatal hernia that compresses the heart and impairs respiratory function (8). The present patient had exertional dyspnea for the previous 10 years. Thus, the exertional dyspnea might have been partially caused by the hiatal hernia.

A previous study showed that pericarditis due to gastrocardiac fistula results in ST elevation (9). However, in the present case, the ST segment and T wave changes might be caused by change of direct or indirect pressure exerted on the heart without a gastrocardiac fistula. $\mathrm{T}$ wave inversion in leads I, II, III, $\mathrm{V}_{\mathrm{L}}, \mathrm{V}_{\mathrm{F}}$ and $\mathrm{V}_{3}-\mathrm{V}_{6}$ on admission similar to that observed in ACS might lead to misdiagnosis. Furthermore, aortic aneurysm, cardiac tumor, and esophageal tumor were considered as the possibilities, because echocardiography showed a mass just behind the heart. However, CT ruled out these possibilities. The low potassium value $(3.5 \mathrm{mEq} / \mathrm{ml})$ and the use of famotidine might be the cause of the prolonged QTc in the electrocardiogram on admission. The cause of the $\mathrm{T}$ wave inversion in the electrocardiogram on admission is still unclear. Fortunately, we were able to distinguish this condition from ACS by echocardiography and by electrocardiographic changes before and after drainage. In addition, the levels of troponin $\mathrm{T}$ and creatinine kinase-MB isoenzyme were within the normal limits on serial determinations. The cause of the ST elevation after drainage of the intragastric fluid might have been pericarditis, because pericardial effusion was visualized with echocardiography 3 days after admission. The $\mathrm{T}$ wave inversion vanished after drainage of intragastric fluid suggesting that it was in some way related to cardiac compression. Moreover, the ST elevation and inflammation diminished without the appearance of $\mathrm{T}$ wave inversion in several days. Thus, it is possible that the cause of pericarditis might be the rapid reduction of cardiac compression by drainage. Furthermore, the electrocardiographic changes might be improved by the operation that relieved the cardiac compression. However, the etiology of pericarditis is still unclear.

In 1904, Borchardt described the clinical findings in patients with gastric volvulus due to organoaxial rotation of the stomach within the paraesophageal hiatal hernia sac (10). These symptoms included chest pain, retching without the ability to vomit, and the inability to pass a nasogastric tube beyond the intrathoracic stomach. In the present case, the patient was able to vomit and pass a nasogastric tube, because gastric volvulus was absent. Thus, the drainage of intragastric fluid relieved the symptoms. In addition, giant hiatal hernias have also been associated with several more esoteric complications including colonic obstruction (11), hemorrhagic pancreatitis (12), gastrobronchial (13), and gastrocardiac fistula (14). Fortunately, the present patient did not have these critical complications, and surgical repair of the hiatal hernia was possible.

Kounis and co-workers showed that patients with hiatal hernia had some electrocardiographic alternation, and increased central venous and pulmonary capillary wedge pressures (7). Echocardiography on admission showed compression of the IVC by the giant hiatal hernia in addition to cardiac compression. In fact, the present patient had both facial and leg edema. A previous report showed that the electrocardiographic alternation disappeared, and the pressures returned to normal after correction of the condition (7). In our patient, facial, leg edema and the electrocardiographic changes improved after the operation for the hiatal hernia. Thus, the electrocardiographic changes and the edema may have been caused by direct or indirect pressure exerted on the heart and great vessels.

The increased BNP level on admission might indicate diastolic dysfunction due to cardiac compression, because it has been shown that BNP levels increase in patients with diastolic dysfunction (15). Furthermore, natriuretic peptides are released in response to increased intracardiac pressure (16). The increase in direct or indirect pressure exerted on the global surface of the heart might cause the increased intracardiac pressure (7). Based on theses previous reports, it is possible to consider that the cause of increased BNP levels 


\section{HOKAMAKI et al}

might be cardiac compression by giant hiatal hernia.

In summary, compression of the heart by a giant hiatal hernia showed dynamic electrocardiographic changes and edema. The electrocardiographic abnormalities and edema were resolved after surgical correction of the hiatal hernia.

Acknowledgements: This study was supported in part by a Research Grant 14C-4 for Cardiovascular Disease from the Ministry of Health and Welfare, a Grant-in-aid for Scientific Research (B-15390248, C2-13670727, 14770318) from the Ministry of Education, Science and Culture in Japan, and a Smoking Research Foundation Grant for Biomedical Research, Japan Cardiovascular Research Foundation, Japan Heart Foundation/Pfizer Grant for Research on Hypertension, Hyperlipidemia and Vascular Metabolism, Tokyo, Japan.

\section{References}

1) Tibbling L. Angina-like chest pain in patients with oesophageal dysfunction. Acta Med Scand Suppl 644: 56-59, 1981.

2) Roberts R, Henderson RD, Wigle ED. Esophageal disease as a cause of severe retrosternal chest pain. Chest 67: 523-526, 1975.

3) Monro JL, Nicholls RJ, Hately W, Murray RS, Flavell G. Gastropericardial fistula - a complication of hiatus hernia. Br J Surg 61: 445447, 1974.

4) Steiner E, Tauscher T, Buchberger W, Waldenberger W. Gastropericardial fistula following transthoracic fundoplication. Wien Klin Wochenschr 101: 746-749, 1989 (in German).

5) Bernasconi M, Brambilla G, Fiorista F, et al. Auriculo-ventricular block upon swallowing. Schweiz Med Wochenschr 115: 1857-1860,
1985 (in French).

6) Akdemir I, Davutoglu V, Aktaran S. Giant hiatal hernia presenting with stable angina pectoris and syncope-a case report. Angiology 52: 863$865,2001$.

7) Kounis NG, Zavras GM, Kitrou MP, Soufras GD, Constantinidis K. Unusual electrocardiographic manifestations in conditions with increased intrathoracic pressure. Acta Cardiol 43: 653-661, 1988.

8) Ito $\mathrm{H}$, Kitami M, Ohgi S, et al. Large hiatus hernia compressing the heart and impairing the respiratory function: a case report. J Cardiol 41: 29-34, 2003 (in Japanese).

9) Mukai M, Ninomiya T, Ochi N, Hamada M. An 80-year-old female with pneumopericardium due to gastric perforation. Nippon Ronen Igakkai Zasshi 32: 123-127, 1995 (in Japanese).

10) Borchardt M. Zur pathologie und therapie des magenvolvuls. Arch Klin Chir 74: 243-246, 1904 (in German)

11) Wichterman K, Geha AS, Cahow CE, Baue AE. Giant paraesophageal hiatus hernia with intrathoracic stomach and colon: the case for early repair. Surgery 86: 497-506, 1979.

12) Kafka NJ, Leitman IM, Tromba J. Acute pancreatitis secondary to incarcerated paraesophageal hernia. Surgery 115: 653-655, 1994.

13) Chong WK, Constant OC. Gastrobronchial fistula. Clin Radiol 41: 141-142, 1990.

14) Standaert R, Dutro JA. Development of ventriculogastric fistula after left ventricular aneurysmectomy. Am Heart J 128: 1250-1252, 1994.

15) Lubien E, DeMaria A, Krishnaswamy P, et al. Utility of B-natriuretic peptide in detecting diastolic dysfunction: comparison with Doppler velocity recordings. Circulation 105: 595-601, 2002 (Erratum in: Circulation 106: 387, 2002).

16) Struthers AD. Prospects for using a blood sample in the diagnosis of heart failure. QJM 88: 303-306, 1995. 